\title{
Mucormycosis: A Deadly Complication of COVID-19 and Diabetes
}

\author{
Manu Jain ${ }^{1}$, Puneet Rijhwani ${ }^{2}$, Ashish Jain $^{3}$, Anchin Kalia $^{4}$, Dinesh Yadav ${ }^{5}$, Ambika Tyagi $^{6}$
}

\begin{abstract}
Mucormycosis is a fungal infection, caused by fungi of the order Mucorales of subphylum Mucoromycotina. It is an aggressive, life-threatening infection that is common in immunocompromised patients and diabetics. COVID-19 infection and its treatment renders the patient severely immunocompromised and can increase the risk of such secondary infections. Here, we present a case of a 62-year-old man, a known case of diabetes who was recently diagnosed as COVID-19 positive. He then complained of left eye swelling and pain, with chemosis while being on steroids and remdesivir for the COVID-19 pneumonia. He was later diagnosed with orbital cellulitis, with a possible fungal infection which was later confirmed microbiologically. The patient was started on liposomal amphotericin B and high-grade antibiotics for orbital cellulitis. The patient later succumbed to sepsis-related complications. We conclude that prompt identification of symptoms and early initiation of therapy is necessary to have a better outcome in such cases, also this case can help us be more vigilant in diabetic patients with COVID-19, in whom therapeutic agents like steroids and monoclonal antibodies should be given at lowest recommended doses.

Keywords: COVID-19, Diabetes, Mucormycosis.

Journal of Mahatma Gandhi University of Medical Sciences \& Technology (2021): 10.5005/jp-journals-10057-0149
\end{abstract}

\section{BACKGROUND}

Mucormycosis is a fungal infection, caused by fungi of the order Mucorales of subphylum Mucoromycotina. It can manifest with different syndromes, particularly acute and life-threatening commonly in diabetics and immunocompromised patients. Its most common presentation is rhino-orbito-cerebral mucormycosis. COVID-19 is a novel coronavirus infection, which affects the immune system rendering the patient vulnerable to secondary infections. Especially in patients with preexisting diseases like diabetes mellitus.

\section{Case Description}

A 62-year-old, male patient, known case of diabetes mellitus presented to the emergency department with complaints of fever and non-productive cough for 3 days and shortness of breath for 1 day. His COVID-19 RT-PCR was positive and HRCT was consistent with COVID-19 with CORADS 5 and CT severity score of $17 / 25$. At the time of presentation, the patient was tachypneic and tachycardic with a respiratory rate of $34 /$ minute and heart rate of $124 /$ minute. He had an oxygen saturation of $88 \%$ on room air and required oxygen support. The patient was evaluated and admitted accordingly to COVID ICU. Routine investigations were ordered and appropriate treatment was started according to protocol. The patient was started on methylprednisolone $2 \mathrm{mg} / \mathrm{kg} /$ day, remdesivir, empirical antibiotic, and symptomatic management (Figs 1 to 5).

The patient did now show improvement while being on this treatment till 6 days of admission, further investigations were ordered. The chest roentgenograph was suggestive of worsening ARDS. The patient was persistently tachypneic and had worsening respiratory failure and the patient was initiated on non-invasive ventilatory support. Serum interleukin-6 levels were $136.2 \mathrm{pg} / \mathrm{mL}$. Because of worsening respiratory failure and high IL- 6 levels, the patient received tocilizumab. The patient's condition improved by day 12 , and the patient was shifted out of the ICU.
1,2,4,6 Department of General Medicine, Mahatma Gandhi Medical College and Hospital, Jaipur, Rajasthan, India

${ }^{3}$ Department of Respiratory Medicine, Mahatma Gandhi Medical College and Hospital, Jaipur, Rajasthan, India

${ }^{5}$ Department of Surgical Oncology, Mahatma Gandhi Medical College and Hospital, Jaipur, Rajasthan, India

Corresponding Author: Manu Jain, Department of General Medicine, Mahatma Gandhi Medical College and Hospital, Jaipur, Rajasthan, India, Phone: +91 8800578870, e-mail: doc.manujain@gmail.com

How to cite this article: Jain M, Rijhwani $P$, Jain A, et al. Mucormycosis: A Deadly Complication of COVID-19 and Diabetes. J Mahatma Gandhi Univ Med Sci Tech 2021;6(1):35-38.

Source of support: Nil

Conflict of interest: None

On day 13 , the patient complained of pain and swelling in the left eye. On examination, there was swelling around the left eye, which was tender along with chemosis. Ophthalmology consultation was taken, and the patient was started on moxifloxacin and ketorolac eye drops, along with oral linezolid.

The swelling in the left eye progressed over the next few days, and the patient started to have blackish discoloration over the left eyelids. On bedside examination, the right eye was normal, but the left eye was chemosed, the extraocular movements were restricted in all planes. The pupils were reactive, but the patient had decreased vision from the left eye. Ophthalmology consultation was taken, and escalation of topical and intravenous antibiotics was done.

The swelling in the left eye increased, with blackish discoloration on the medial side with a formation of a clot. Further investigations and escalation of antibiotics were planned, but as he tested negative on day 20 , he refused to stay for further management.

After getting discharged, the patients' general condition worsened at home. The patient started having swelling around the right eye, with decreased vision from the right eye. The patient was 

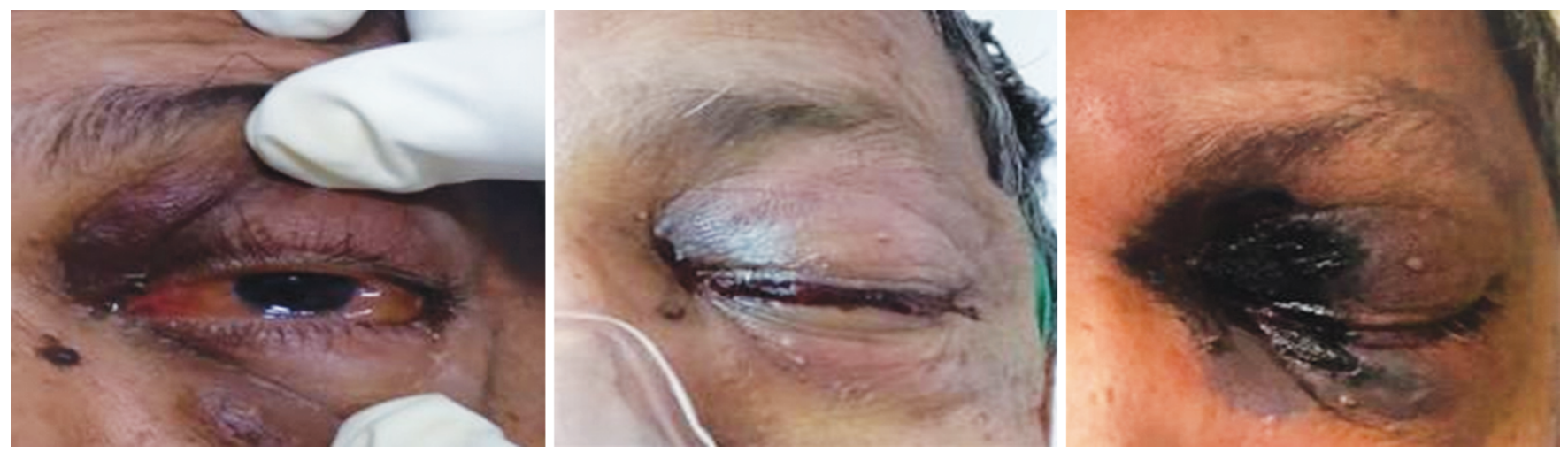

Fig. 1: Progressive changes as seen in the left eye of the patient from swelling to eschar formations
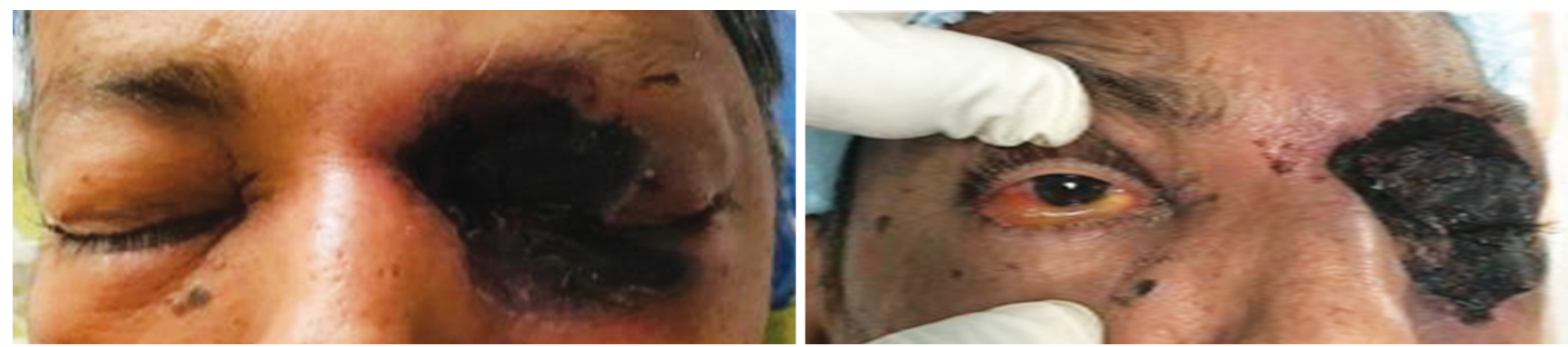

Fig. 2: Preoperative pictures showing the progression of eschar over the left eye and development of swelling over the right eye

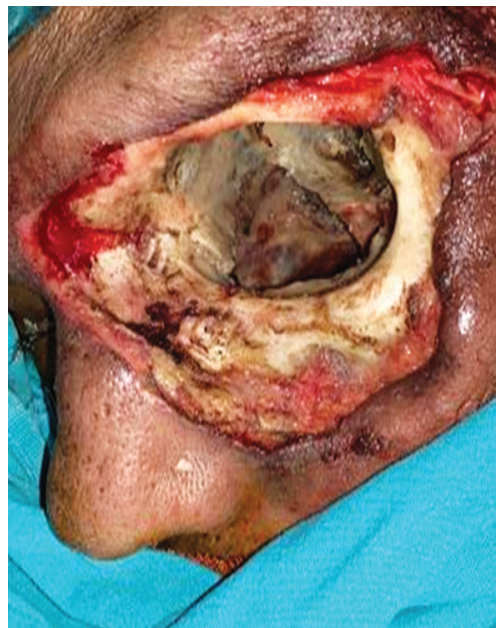

Fig. 3: Intraoperative picture showing widespread involvement of orbit and surrounding tissue

then admitted to another hospital with complaints of decreased vision in the right eyes, followed by irrelevant talks and altered sensorium. MRI brain was done, which was suggestive of patchy areas of altered signal intensity appearing hyperintense on FLAIR and restriction diffusion seen in the left cerebral hemisphere in the basifrontal and anterior temporal regions. With subdural collection s/o cerebritis, and evidence of pansinusitis with left peri- and intraorbital collections. CECT brain and PNS were advised which was suggestive of pansinusitis with left orbital cellulitis. Deformed left optic globe with thrombosed superior ophthalmic vein and features of left frontotemporal cerebritis.

The patient was then referred to Mahatma Gandhi Hospital, where he was admitted to the General medicine department.

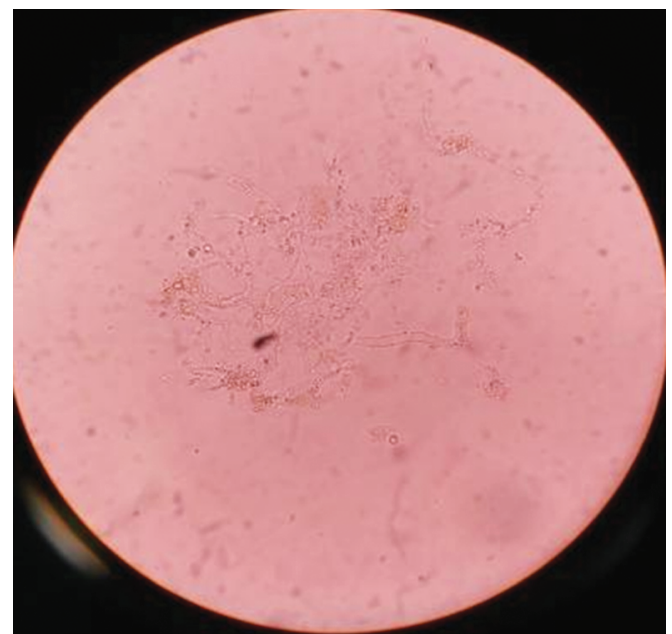

Fig. 4: $\mathrm{KOH}$ mount of the scraping taken from the eschar showing broad, aseptate, hyaline hyphae

The patient was provisionally diagnosed post-COVID pneumonia, left orbital cellulitis, left temporal cerebritis, CVST with possible bacterial or fungal etiologies.

The patient was started on empirical antibiotics and liposomal amphotericin B at $5 \mathrm{mg} / \mathrm{kg} /$ day. Investigations were sent, including Gram stain, $\mathrm{KOH}$ mount, bacterial and fungal cultures from the eschar site which was suggestive of broad, aseptate, hyaline hyphae.

Ophthalmology reference was taken for the same, and E/d voriconazole and Syp posaconazole were added. The patient was observed for response to antifungal therapy. Along with medical management, the patient was deemed to be a candidate for surgical debridement after careful discussion with ophthalmology and ENT 


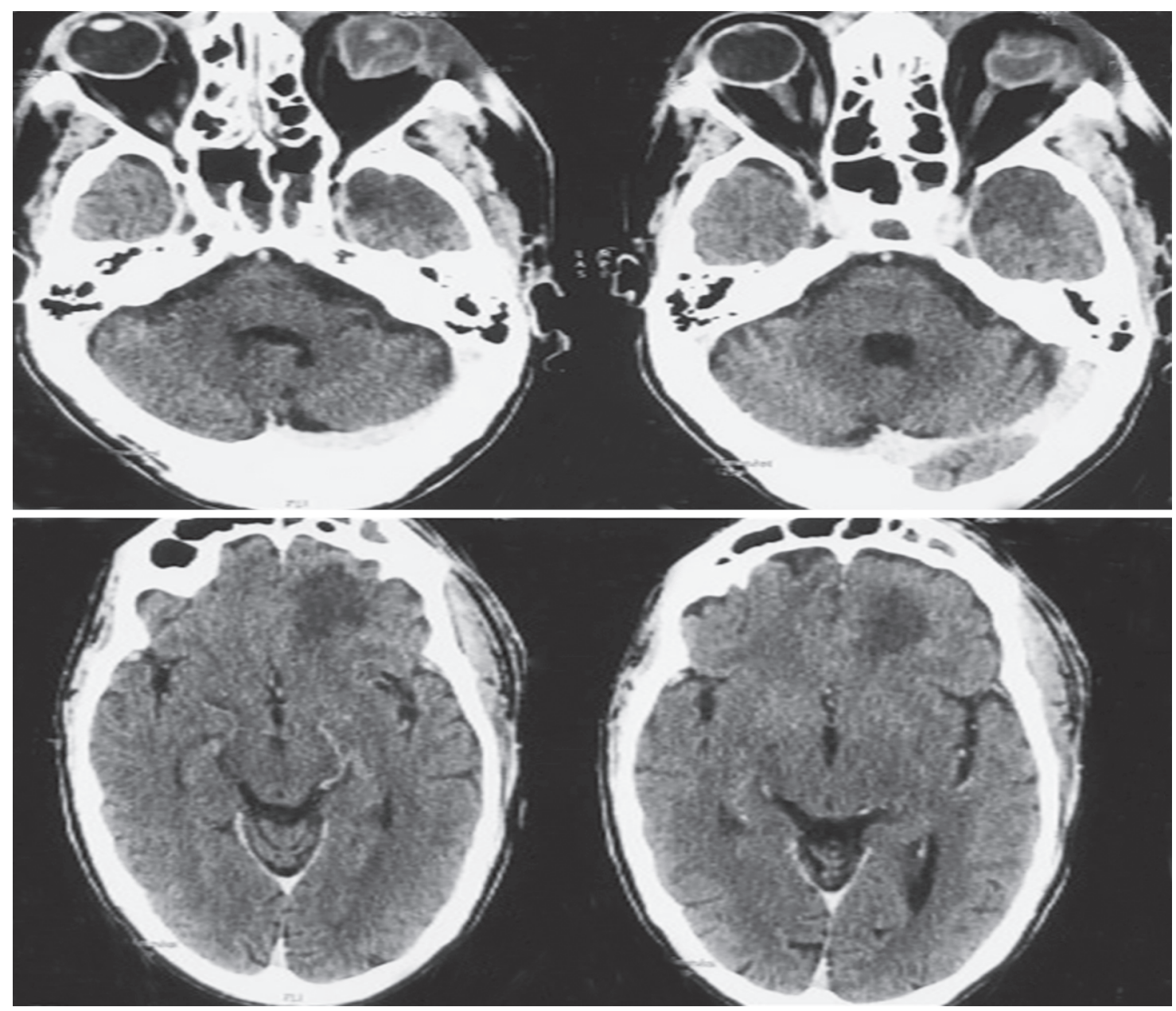

Fig. 5: CECT head axial section showing the left orbital cellulitis, deformity in left orbit with left frontotemporal cerebritis

departments. The patient underwent an exenteration of the left orbit with maxillary sinuses.

Postoperatively, the patient was managed in ICU. But later succumbed to widespread sepsis and its sequelae.

\section{Discussion}

Mucormycosis has been classically associated with conditions like diabetes mellitus, hematological malignancies, and hematopoietic cell transplantation. ${ }^{1}$ It is one of the most important and deadly secondary infections in diabetes. Rhino-orbito-cerebral mucormycosis is the commonest presentation of Mucormycosis. Recently with the increasing cases of COVID-19, we have observed a high incidence of mucormycosis in patients with active COVID-19 or post-recovery from COVID-19.

At present, the guidelines in India recommend intravenous methylprednisolone $0.5-1 \mathrm{mg} / \mathrm{kg} /$ day in moderate cases and $1-2 \mathrm{mg} / \mathrm{kg} /$ day in severe cases. ${ }^{2}$ The guidelines warn against the risk of developing secondary infections. ${ }^{3}$

Many factors including preexisting conditions, such as diabetes mellitus, old respiratory illnesses, risk of hospital-acquired infections, use of immunosuppressive agents, and the immune effects of COVID-19 itself, may lead to the development of secondary infections. ${ }^{4}$ Therefore, COVID-19 may itself increase the risk of developing a secondary infection by the secondary changes it causes in the organs or by the immune dysregulation associated with it. The reduced number of T lymphocytes, CD4+ and CD8+ specifically may alter patients' innate immunity.

Mucormycosis can also be seen in patients with COVID-19, without preexisting diabetes mellitus. This is in part because of the steroids, not just because of the immunosuppression but also because of the transient hyperglycemia. We have noticed transient hyperglycemia in patients receiving therapeutic doses of steroids even with normal HbA1c values. An uneven level of blood sugar weakens many aspects of innate and adaptive immune response to viral and possible secondary bacterial infections.

Patients with diabetes mellitus have increased severity of COVID-19 disease and worse outcomes. ${ }^{5}$

Along with medical management, there is a need for the incorporation of surgical management as well. The European Confederation of Medical Mycology Mucormycosis Guidelines strongly suggest an early surgical treatment to remove the infected tissue in addition to systemic antifungal treatment. ${ }^{6}$ Just like in our case, the patient was taken for exenteration of the left orbit and maxillary sinuses to remove the infected and infarcted tissue.

The patient we described, presented to us with complaints specific and limited to a COVID-19 infection and had no complaints or symptoms suggestive of a hidden mucormycosis infection. The 
signs of an orbital infection only started to develop around day 13 of his admission. The patient was a known case of diabetes mellitus and was taking homeopathic medications for the same. He had no record of treatment or any kind of follow-up for the management. During admission, the patient received broadspectrum antibiotics, high potency steroids, and monoclonal antibodies for COVID-19 infection. All these factors increased the risk for the development of a fungal infection, alongside the immune alterations caused by COVID. Therefore, in our case, either a previously undiagnosed infection may have aggravated or a new infection may have developed as a result of a combination of all these factors.

\section{Conclusion}

There is a significant and urgent need for increased vigilance regarding the screening and treatment of secondary infections during COVID-19, especially in patients with preexisting comorbidities. These might in part be due to the immune dysregulation caused by COVID-19. Moreover, the use of broadspectrum antibiotics, high potency steroids, and monoclonal antibodies may lead to the development of a new secondary infection or exacerbation of an old one. Especially, opportunistic fungal infections.

Physicians should be aware of the possibility of secondary infections, including secondary fungal infections. The use of therapeutic agents like steroids and monoclonal antibodies should be at the lowest recommended doses, with adequate risk/benefit analysis to reduce the chances of unwanted effects.

\section{References}

1. Roden MM, Zaoutis TE, Buchanan WL, et al. Epidemiology and outcome of zygomycosis: a review of 929 reported cases. Clin Infect Dis 2005; 41(5):634-653. DOI: 10.1086/432579.

2. Clinical management protocol for COVID-19. [Jul;2020]; https://www. mohfw.gov.in/pdf/ClinicalManagementProtocolforCOVID19.pdf 2020 [Ref list].

3. COVID-19 Treatment Guidelines Panel. Coronavirus disease 2019 (COVID-19) treatment guidelines. National Institutes of Health. [Jul;2020]; https://www.covid19treatmentguidelines.nih.gov/ 2020 [Ref list].

4. Lega S, Naviglio S, Volpi S, et al. Recent insight into SARS-CoV2 immunopathology and rationale for potential treatment and preventive strategies in COVID-19. Vaccines 2020;8(2):224. DOI: 10.3390/vaccines8020224.

5. Derouiche S, Cheradid T, Abdelmalek D, et al. Effect of COVID-19 infection on the immune system and risk of developing diabetes complications: a review. J Pharm Care 2020;8(3):133-139.

6. Cornely OA, Alastruey-Izquierdo A, Arenz D, et al. Global guideline for the diagnosis and management of mucormycosis: an initiative of the European Confederation of Medical Mycology in cooperation with the Mycoses Study Group Education and Research Consortium. Lancet Infect Dis 2019;19(12):e405-e421. DOI: 10.1016/S14733099(19)30312-3. 